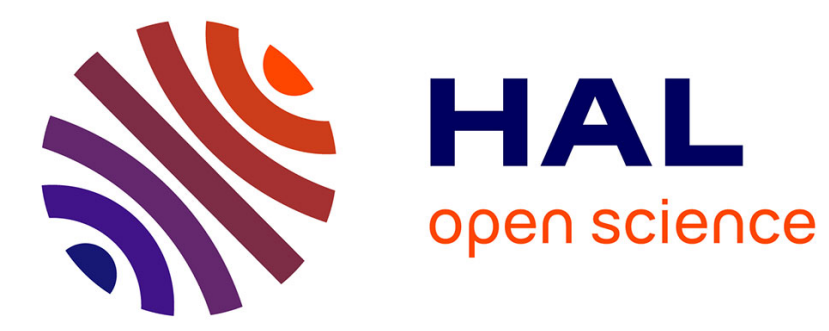

\title{
L'autonomie des collégiens présentant un retard mental : ses conditions de possibilité en éducation physique et sportive

\author{
Jean-Pierre Garel
}

\section{- To cite this version:}

Jean-Pierre Garel. L'autonomie des collégiens présentant un retard mental: ses conditions de possibilité en éducation physique et sportive. Reliance, 2007, 24, pp.36-48. 10.3917/reli.024.0036 . hal01947603

\section{HAL Id: hal-01947603 \\ https://hal-inshea.archives-ouvertes.fr/hal-01947603}

Submitted on 7 Dec 2018

HAL is a multi-disciplinary open access archive for the deposit and dissemination of scientific research documents, whether they are published or not. The documents may come from teaching and research institutions in France or abroad, or from public or private research centers.
L'archive ouverte pluridisciplinaire HAL, est destinée au dépôt et à la diffusion de documents scientifiques de niveau recherche, publiés ou non, émanant des établissements d'enseignement et de recherche français ou étrangers, des laboratoires publics ou privés. 


\title{
L'autonomie de collégiens présentant un retard mental : ses conditions de possibilité en Éducation physique et sportive
}

\author{
Jean-Pierre Garel \\ Cnefei (Centre national d'études et de formation pour l'enfance inadaptée) \\ Laboratoire Relacs, Université du Littoral Côte d'Opale
}

Conquête participant à l'accomplissement de soi et source légitime du pouvoir des sociétés fondées sur la citoyenneté, l'autonomie de l'individu est un enjeu éducatif majeur, souligné dans les textes officiels de l'Éducation nationale, et un élément central de l'idéologie individualiste actuellement très prégnante, dont témoigne la promotion de comportements appelés à faire de chacun l'acteur et l'auteur de sa propre histoire : «Faire preuve d'autonomie, s'investir dans des projets, manifester son sens des responsabilités, prendre des risques, voilà ce qui nous est demandé. $»^{1}$

La réponse que des personnes en situation de handicap peuvent apporter à cette demande risque d'être décevante en raison de leurs difficultés propres et des contraintes extrinsèques qui les majorent. Pour elles, l'enjeu de l'autonomie ne s'apprécie pas seulement au regard de finalités éducatives valables pour tous et par rapport à une idéologie actuelle. Plus spécifiquement, il s'agit d'un « enjeu existentiel qui questionne l'inscription effective de la différence dans le monde commun $»^{2}$. On comprend donc que l'autonomie soit au cœur des principes de l'action en direction de ces personnes. Elle conditionne leur participation sociale et suppose que la société s'emploie à créer les conditions de son développement. À cet égard, l'école a une responsabilité de premier ordre, que nous questionnerons à propos de l'EPS et d'une population déterminée, envisagée dans un contexte particulier, en nous appuyant sur une étude conduite au collège Pierre Mendès France, à Riom $(63)^{3}$ : dans quelle mesure l'enseignement de cette discipline peut-il contribuer à l'autonomie d'adolescents présentant un retard mental et qui sont scolarisés dans un collège ordinaire ?

\footnotetext{
1 Kokoreff M. et Rodriguez J. (2005), « Une société de l'incertitude », « Sciences Humaines, Hors-série n50, p.7.

${ }^{2}$ Pierron J.-P. (2007), « L'autonomie dans la dépendance : le libre choix des personnes en situation de handicap », Bulletin d'informations du CREAI Bourgogne, $\mathrm{n}^{\circ} 266, \mathrm{p} .10$.

3 Voir Garel J.-P. (2001), Des collégiens parmi les autres. L'intégration en éducation physique et sportive d'élèves présentant un handicap mental, Doc. audiovisuel, 40', Édit. de l'INS HEA.
} 


\section{La reconnaissance des collégiens présentant un retard mental}

L'autonomie d'un individu demande qu'il se reconnaisse et soit reconnu comme un sujet capable d'agir de lui-même et disposant de droits. La reconnaissance de son identité en est un préalable.

\section{1. Reconnaître l'identité}

L'identité que chacun se reconnaît, l'histoire qu'il se raconte sur ce qu'il est, la représentation qu'il se fait de lui-même, en un mot l' « identité pour soi », est liée à l' « identité pour autrui » En d'autres termes, elle appelle un regard extérieur, sollicité dès la première étape du parcours de la reconnaissance tracé par Paul Ricœur ${ }^{5}$, celle de l'identification de l'individu, de sa distinction parmi les autres.

Quand on cherche des références aux élèves présentant des troubles importants des fonctions cognitives $^{6}$ dans les documents destinés aux enseignants d'EPS en poste dans les établissements scolaires ordinaires, on est surpris de constater leur quasi-absence, particulièrement si on circonscrit la recherche aux sources institutionnelles de l'Éducation nationale : parmi les trentedeux sites académiques dédiés à cette discipline sur le web, deux seulement évoquent succinctement ces élèves. C'est sans commune mesure avec la documentation pédagogique que l'on peut y trouver concernant les élèves présentant une déficience motrice ou visuelle. Et dans un récent dossier élaboré par le Groupe ressource pour l'intégration des élèves à besoins spécifiques de l'Académie de Grenoble, publié dans la revue EP\&S, pas une ligne n'est consacrée à cette population ${ }^{7}$.

Pour reprendre le titre du rapport présenté lors de la «Journée européenne des Personnes handicapées ", le sept décembre 1995, on a affaire à des "citoyens invisibles "; ce qui est étrange au regard de la présence effective des élèves identifiés comme porteurs d'une « atteinte intellectuelle ou mentale» dans les statistiques de l'Éducation nationale. Par rapport à l'ensemble des élèves en situation de handicap qui sont scolarisés dans des établissements scolaires ordinaires, ils sont en effet très nombreux : dans le premier degré, ils constituent $89 \%$ des effectifs de Clis (Classe d'intégration scolaire) et 39\% des intégrations individuelles; en collège, ils représentent $74 \%$ des élèves d'UPI (Unité pédagogique d'intégration) et $14 \%$ des intégrations individuelles, et, en lycée, respectivement $43 \%$ et $12 \%$.

Comment comprendre qu'ils soient ignorés ? Un premier élément d'explication réside peut-être dans une focalisation sur l'évaluation, notamment celle qui est requise aux examens : CAP,

\footnotetext{
${ }^{4}$ Goffman E. (1975), Stigmates. Les usages sociaux du handicap, Paris, Ed. de Minuit.

${ }^{5}$ Ricoeur P. (2004), Parcours de la reconnaissance, Paris, Stock

${ }^{6}$ Depuis la circulaire $n^{\circ} 2001-035$ du 21 février 2001, « handicap mental» fait place, dans les textes officiels de l'Éducation nationale, à la dénomination « troubles importants des fonctions cognitives ». Selon la circulaire, ces troubles peuvent tenir à « un retard mental global, à des difficultés cognitives électives, à des difficultés psychiques graves... «

${ }_{7}^{7}$ «Une intégration réussie en EPS : utopie ou réalité ? » (2006), Dossier, Revue EP\&S., n 139, p. 15-51. Ce dossier se décentre toutefois des déficiences motrices et sensorielles en évoquant l'EPS avec des adolescents phobiques.

${ }^{8}$ Repères et références statistiques, MEN, Édition 2006, p. 27, Disponible sur : http://media.education.gouv.fr/file/31/8/2318.pdf
} 
BEP, baccalauréat. L'EPS a construit sa légitimité dans l'univers scolaire en partie sur la notation à laquelle elle donne lieu à cette occasion, y trouvant de quoi conforter son statut de discipline d'enseignement à part entière, obligatoire pour tous, plus ou moins à l'abri des tentatives de soustraction à la tutelle du ministère de l'Éducation nationale, qui viseraient à en faire une pratique sportive comme les autres, confiée à des personnels moins formés et plus économiques pour le budget de l'État.

Le souci de l'évaluation en EPS est particulièrement prégnant dans le second degré. Quand on considère les productions relatives aux élèves à besoins spécifiques en EPS, disponibles sur les sites académiques, on remarque l'omniprésence de l'évaluation, qui constitue d'ailleurs bien souvent une demande prioritaire d'information et de formation des enseignants concernant ce type d'élève. Au point que l'on peut se demander si un enseignement qui n'ouvre pas, à plus ou moins long terme, sur la perspective d'une évaluation à un examen, comme c'est a priori le cas des adolescents présentant un retard mental, ne s'en trouve pas dévalorisé et négligé. L'éducation physique de ces jeunes ne suscite pas d'intérêt. À notre connaissance, elle n'est jamais un thème central de la formation continue des professeurs au niveau des Académies. Les élèves que l'on n'a pas à évaluer aux examens n'auraient-ils donc pas d'existence ?

Une seconde explication possible au manque d'intérêt qui leur est porté, dans le domaine de l'EPS, tient peut-être aussi à une absence d'altération physique manifeste. En effet, une incapacité est souvent inférée de la visibilité du handicap. Pourquoi se mobiliser pour faire face à des difficultés et des personnes qui sont ignorées ? L'aspect souvent peu spectaculaire des problèmes physiques d'élèves avec un retard mental, par rapport à d'autres handicaps, ne devrait pas conduire à les sous-estimer, pas plus que les problèmes affectifs ou relationnels qui sont également parfois présents. Les connaissances des troubles pouvant accompagner tel ou tel type d'altération mériteraient d'être introduites dans la formation des enseignants. Elles sont précieuses en ce qu'elles attirent l'attention du professeur sur des difficultés qu'il est susceptible de rencontrer et dans la mesure où elles ne construisent pas chez lui des représentations figées. On doit en effet éviter d'assigner les personnes à des catégories (trisomie, autisme...) dont tous les membres seraient prétendument identiques et fondamentalement différents des personnes « normales ». Même si beaucoup d'individus faisant l'objet d'une même désignation partagent des caractéristiques semblable, ce qui les distingue des autres est moins fort que ce qui les réunit. En l'occurrence, nous considérons les élèves de l'UPI de Riom davantage comme des collégiens que des «handicapés». Et les marques communes, sinon les stigmates, qui les éloignent de la norme ne sont pas fatalement dans leur nature : le surpoids, ou l'obésité, de l'adolescent trisomique n'est pas inévitable. Reconnaitre l'identité des collégiens en situation de handicap mental appelle une connaissance nuancée, dénuée de préjugés, de leurs possibles difficultés ainsi que de leurs capacités.

\section{2. Reconnaître les capacités}

La seconde étape de la reconnaissance abordée par Paul Ricœur porte sur la "capacité de faire arriver des événements dans l'environnement physique et social ${ }^{9}{ }^{9}$, une dimension qui approfondit l'identité du sujet, puisqu'il est notamment définissable par ce dont il est capable et ce qui lui est imputable, et qui est fondamentale dans une réflexion sur l'autonomie. En effet,

\footnotetext{
${ }^{9}$ Ricoeur P., op. cit., p. 199.
} 
pour éviter l'emprise de dépendances aliénantes, exprimer ses choix, faire valoir ses droits, s'engager dans des apprentissages émancipateurs en acceptant le risque de ne pas toujours réussir, le sujet doit avoir suffisamment confiance en soi et par conséquent reconnaître ses capacités. Or les capacités sont occultées dès lors qu'elles ne se donnent pas à voir faute d'être sollicitées activement, et des sollicitations ambitieuses nécessitent de croire en l'éducabilité des élèves en situation de handicap.

Cette croyance ne relève pas d'un pari, au sens d'un espoir que la chance sourit, mais du constat maintes fois établi de réussites qui tiennent moins au hasard qu'à un enseignement adapté fondé sur des exigences élevées. Avancer que les objectifs pédagogiques formulés pour des élèves « handicapés » doivent être « modestes » manque d'ambition. Certes ils ne sont pas toujours identiques à ceux qui sont prévus pour les autres élèves, mais ils doivent être envisagés de façon à permettre à chacun de réaliser le meilleur de lui-même. Dire, comme nous l'avons entendu, à propos d'un élève « intégré » qu' « il n'a pas appris grand-chose mais (qu') il était bien avec les autres », et s'en satisfaire, traduit une conception de l'intégration qui fait d'une socialisation mal comprise un objectif envahissant : socialisation et accomplissement personnel sont intimement liés et supposent des apprentissages, y compris pour devenir plus autonome.

Les capacités sociales sont l'objet d'une reconnaissance particulière, l'estime sociale. Peuvent notamment y concourir les activités qui relèvent de pratiques sociales valorisées et valorisantes, susceptibles d'instaurer « du lien social et des modalités d'identité qui s'y rattachent ${ }^{10}$. De ce point de vue et pour ce qui est de l'EPS, les activités physiques de nature sportive et artistique sont préférables à celles qui privilégient une réhabilitation orientée par une conception biomédicale de la personne. L'enseignement n'a pas pour vocation de soigner, même si, en l'occurrence, il peut avoir des bénéfices thérapeutiques.

La reconnaissance des capacités passe par l' «externalisation » de ce que réalise le sujet ${ }^{11}$, la présentation de son «œuvre», source de fierté. En clair, il est intéressant que le résultat de l'activité conduite par l'élève soit donné à voir pour être reconnu par son enseignant et ses camarades, et qu'il laisse si possible une trace : sur une fiche, un cahier, à partir d'une photo, etc. Autant d'éléments objectifs qui ressortissent à l'évaluation et fournissent à l'adolescent le support objectif d'une prise de conscience de ses progrès, dont d'autres personnes, notamment ses proches, pourront prendre connaissance et sur lequel pourra se fonder une reconnaissance gratifiante $^{12}$.

L'évaluation d'adolescents en situation de handicap serait davantage un vecteur de mésestime de soi et de découragement que de reconnaissance si elle n'était pas adaptée à leurs possibilités. Au collège Pierre Mendès France, les élèves de l'UPI ne sont pas obligatoirement évalués par rapport aux critères appliqués aux autres collégiens. Il y a là de quoi ébranler la représentation que des enseignants se font de l'égalité. Certains sont choqués que des candidats aux examens ne soient pas soumis aux épreuves «normales », ainsi que l'autorisent, et même le préconisent les textes officiels. Dans le dossier de la revue EP\&S évoqué précédemment, un professeur

\footnotetext{
${ }^{10}$ Ricoeur P., ibid., p. 202.

${ }^{11}$ Bruner J. S. (1996). L'éducation, entrée dans la culture, Paris, Retz, p. 39.

${ }^{12}$ Garel J.-P. (2006), « La reconnaissance de la capacité d'agir sur et par un corps altéré, enjeu de la construction du sujet », Éduquer, Paris, L'harmattan, p. 61-71.
} 
s'interroge : «N'est-ce pas un problème pour l'évaluation que ces activités n'appartiennent pas à la liste nationale des épreuves évaluables en Terminale ? ». La question, qui ne concerne pas les élèves présentant un retard mental puisque, a priori, ils ne sont guère concernés par ces

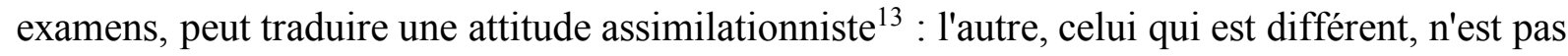
perçu dans sa singularité et doit donc être traité comme tout le monde. A contrario, une attitude différentialiste, exagérément attentive aux différences, pourrait se manifester par des réticences à l'égard d'une pratique mixte, au prétexte que les élèves « handicapés » sont trop singuliers pour être avec les autres en EPS, et par un manque d'exigence dans l'évaluation comme dans les autres aspects de l'enseignement ${ }^{14}$.

\section{3. Reconnaître les droits}

Il ne suffit pas d'avoir acquis des capacités reconnues par soi-même et par les autres si les activités dans lesquelles on pourrait les exercer sont inaccessibles. Il faut aussi reconnaître le droit, pour tous les enfants et adolescents en situation de handicap, d'accéder à l'enseignement de l'EPS, parmi les élèves ordinaires autant que possible, et d'y trouver les conditions d'une pratique optimale au regard des bénéfices attendus. Encore faut-il que la proclamation de ce droit ne soit pas que formelle, qu'elle s'accompagne de droits garantissant cet accès. Il est inadmissible que des élèves soient dispensés d'éducation physique sans raison valable, que certains n'aient pas cours à cause de réticences d'enseignants ou que l'enseignement qu'ils reçoivent ne soit pas adapté, qu'un professeur doive, sans formation et sans soutien, gérer un groupe-classe devenu trop hétérogène, que les heures d'enseignement allouées soient insuffisantes, etc.

Outre l'engagement des partenaires directs du professeur d'EPS (parents, personnel administratif du collège, médecin scolaire, infirmière, inspecteur...), et sans qu'il faille dans tous les cas des textes législatifs supplémentaires, la participation d'élèves à besoins spécifiques aux cours d'EPS peut appeler, autant que de besoin, des mesures qui relèvent de niveaux de responsabilité différents, par exemple : la présence de personnels secondant le professeur durant les cours (auxiliaire de vie scolaire, enseignant spécialisé....) et, plus généralement, des personnes et des dispositifs ressources; des programmes d'activités physiques et des emplois du temps adaptés ; une organisation des enseignants d'EPS du collège qui assure la continuité et la cohérence de l'enseignement durant la scolarité de l'élève ; une dotation horaire globale de l'établissement qui comporte des heures d'EPS spécifiques; l'information et la formation des personnels concernés...

\footnotetext{
${ }^{13}$ Schnapper D. (1998), La relation à l'Autre. Au coeur de la pensée sociologique, Paris, Gallimard, p. 35-68.

${ }^{14}$ Garel J.-P. (2006), «L'adaptation des APSA à des personnes en situation de handicap : entre droit à la différence et droit à la ressemblance », Actes du forum international de l'éducation physique et du sport, 4-5-6 novembre 2005, Paris, Contre-pied, $\mathrm{n}^{\circ}$ 18, Centre EPS et société.
} 


\section{Un enseignement organisé pour accroître les pouvoirs de l'élève}

Sauf à s'illusionner, la reconnaissance des capacités de l'adolescent implique qu'il en soit effectivement doté. À cet effet, l'enseignement doit être pensé de manière à lui permettre d'acquérir des pouvoirs d'action à sa mesure.

\section{1. Les groupements d'élèves}

L'observation des élèves de l'UPI de Riom en dehors des temps de cours, lors des repas ou des récréations, montre la diversité des relations qu'ils cherchent à établir avec les autres élèves du collège. Si certains privilégient les moments entre soi, ce n'est pas le cas de tous. Il serait imprudent de restreindre l'explication de ces choix différents à des sentiments identitaires tels que les uns se percevraient plutôt comme appartenant à un monde à part, tandis que les autres se reconnaîtraient davantage dans les collégiens "ordinaires». D'une part, le sentiment d'appartenance à la communauté des jeunes de l'UPI peut très bien se conjuguer avec l'aspiration à être intégré dans le groupe de référence, informel, constitué par les adolescents « normaux ». D'autre part, la recherche d'interactions des adolescents de l'UPI avec les autres, pour spontanée qu'elle soit hors des temps de cours, est l'effet d'une construction à laquelle participent de multiples activités communes, organisées au sein de l'établissement scolaire : théâtre, projets périscolaires, ateliers du midi, association sportive, actions de tutorat menées autour de l'enseignement de langues étrangères ${ }^{15}$, et certains cours en commun, dont l'EPS

Dans cette discipline, certains élèves de l'UPI sont en situation d'intégration individuelle parce qu'ils adhérent à ce projet de scolarisation, que leurs compétences sociales sont un facteur favorable, et souvent aussi par ce qu'ils retrouvent en EPS des camarades avec lesquels ils partagent, ou ont partagé, d'autres activités, par exemple au sein d'un atelier de la Segpa. Les capacités physiques sont un critère appréciable mais pas déterminant.

Dans ce collège, c'est la situation d'intégration collective qui est privilégiée : deux heures par semaine, les élèves de l'UPI participent aux cours d'éducation physique au sein d'une classe de cinquième. Par ailleurs, ils ont une heure hebdomadaire d'EPS durant laquelle ils ne sont qu'entre eux et qui permet de prendre en compte leurs spécificités : pratiquer une activité qui s'avère très difficile en situation d'intégration ou une autre qui répond à un besoin particulier.

Lors des cours d'EPS avec les élèves « ordinaires », il ne s'agit pas d'une pratique côte à côte, où les adolescents de l'UPI, engagés dans des activités à part, n'auraient pas d'interaction avec leurs camarades de cinquième, mais bien d'une pratique mixte: les groupes de travail comprennent les uns et les autres, dans les équipes de jeux collectifs comme pour d'autres activités. Pour répondre à un besoin de repères stables chez les élèves, ils peuvent demeurer identiques durant un cycle de cours consacrés à une même acticité. D'autres critères que la mixité pourraient présider à la constitution des groupes, par exemple les affinités entre élèves, l'âge ou le niveau de pratique.

\footnotetext{
${ }^{15}$ Galerin L., Guillot G., Boyer J.-P. (2003), « Apprendre ensemble au collège », in Belmont B. et Vérillon A., Diversité et handicap à l'école Quelles pratiques éducatives pour tous ? Paris, CTNERHI/INRP.
} 


\section{2. Le choix des activités}

Des activités sont privilégiées parce qu'elles répondent aux besoins des élèves de l'UPI, et d'autres sont évitées, dans le cadre d'une pratique mixte, en raison des problèmes qu'elles posent. Ainsi, l'insuffisance de ressources cognitives des adolescents peut les mettre en difficulté dans des activités où la réussite dépend pour beaucoup de leur mobilisation. Les prises d'information et de décision qui sont requises dans la pratique des sports collectifs, par exemple, leur sont d'autant plus difficiles qu'ils doivent agir rapidement lorsqu'ils jouent avec leurs camarades de cinquième, globalement d'un meilleur niveau qu'eux. C'est pourquoi les enseignants d'EPS du collège ont décidé de ne pas proposer, en situation d'intégration, des sports aussi exigeants, sur le plan cognitif, que le basketball. En revanche, un jeu collectif comme la thèque, qui sollicite moins les ressources qui leur font défaut, est retenu dans ce contexte.

De façon générale, la compétition sportive peut être un obstacle à une pratique mixte. Dans le cadre de l'UNSS (Union nationale du sport scolaire), des élèves de l'UPI pratiquent le basketball, mais seulement lors des entraînements au sein de l'établissement, car leur niveau de jeu est insuffisant pour qu'ils soient intégrés dans l'équipe du collège engagée dans un championnat scolaire. Lors des entraînements, ils restent entre eux la plupart du temps, pour ne pas entraver la progression des autres joueurs. L'un des membres de l'équipe le regrette, mais l'enjeu de la compétition est trop fort : « On aimerait bien qu'ils participent avec nous dans les entraînements, mais on n'a pas trop le temps, parce qu'on n'a qu'une heure par semaine ; c'est peu pour faire les entraînements, pour faire après les compétitions »".

\section{3. La différenciation des tâches}

De même que le handicap n'existe pas en soi, indépendamment des situations dans lesquelles il peut se manifester, les difficultés d'un élève en EPS ne sont pas inéluctables. Différencier les contraintes des tâches en fonction de ses ressources personnelles ouvre des perspectives de progrès et de gratifications que l'on n'imaginerait pas a priori. Au basketball, par exemple, on peut accorder à certains joueurs un point si leur tir touche le dessus de l'anneau du panier, interdire à leurs adversaires de défendre trop près d'eux, sur tout le terrain ou lors d'un tir au panier, tolérer une «reprise de dribble», etc. Ces règles de jeu particulières facilitent l'accessibilité de ce sport et permet un meilleur équilibre des forces en présence. L'inégalité des droits propres à une activité vise l'égalité des chances.

D'autres règles sont particulières dans la mesure où elles sortent de l'ordinaire, et non parce qu'elles s'appliquent à quelques-uns seulement: un professeur du collège J. Macé, à Mainvilliers (28), attribue un bonus de quinze points à l'équipe de basketball dont tous les joueurs ont marqué au moins un point. De ce fait, les adolescents « handicapés » osent tirer, d'autant que leurs camarades les conseillent pour se placer sur le terrain, les mettent en position favorable pour agir, etc. Selon l'enseignant, les élèves de l'UPI prennent ainsi « beaucoup de plaisir (...). Ils discutent énormément de leur pratique avec leur instituteur ${ }^{16}$.

16 Témoignage de C. Grissault sur : http://www.ac-orleanstours.fr/eps/eps_adaptee/integration_eleves_UPI_en_EPS.pdf 
L'environnement peut aussi donner lieu à des adaptations. Dans un parcours d'obstacles, il est possible de faire varier leur hauteur et la distance les séparant; au badminton, la surface d'un terrain peut être réduite ; pour un travail d'équilibre, une poutre peut être remplacée par un banc, etc. Le petit matériel, manipulable, peut également être adapté : au mini-tennis, une raquette plus grande et des balles en mousse, plus grosses et de couleur vive, sont susceptibles de favoriser l'habileté. Ces quelques exemples ne sont qu'un aperçu des adaptations possibles ${ }^{17}$, toutes conçues à partir de l'identification des capacités et des difficultés de l'élève, puis d'une modulation des facteurs de difficulté de la tâche.

\section{4. L'attribution de responsabilités}

Être à l'origine d'un choix ou d'une action participe de la responsabilité personnelle et contribue à la conscience d'un pouvoir d'être cause, à une meilleure estime de soi et, in fine, à une autonomie accrue. Cet effet positif peut être renforcé si ce qui est imputable au sujet relève d'un rôle socialement reconnu, dont il a à répondre devant les autres et qui lui vaut un regard positif s'il parvient à l'assumer.

Confier des responsabilités suppose de faire confiance. Lorsqu'on s'adresse à des jeunes ayant des troubles importants des fonctions cognitives, il est légitime de se demander s'ils sont prêts à exercer celles que l'on envisage, mais il faut poser que la responsabilité s'apprend progressivement. Son niveau de difficulté, ainsi qu'un éventuel accompagnement par un tiers, sont à décider en fonction de chaque élève, pour des rôles concernant par exemple la gestion d'une activité : responsable du matériel (mettre en place et ranger...); observateur (au basketball, compter le nombre de tirs réussis, cocher le nom des joueurs qui marquent des points...) ; responsable de l'échauffement précédant une activité ; arbitre (plutôt en coarbitrage); pareur (en gymnastique), etc. Par ailleurs, la pratique effective d'une activité physique peut impliquer des rôles qui contribuent à la réalisation d'un projet commun et qui sont à la portée d'élèves ayant un retard mental. Les activités physiques artistiques (liées à la danse, au cirque...) en offrent l'opportunité.

\section{L'accompagnement de l'autonomisation}

Dans sa conquête de l'autonomie, l'adolescent peut bénéficier des interactions avec son professeur et avec tous ceux, adultes et pairs, qui sont présents pendant les cours d'EPS. Elles méritent donc une attention particulière ${ }^{18}$.

17 Garel J.-P. (2003), «Elèves en situation de handicap : la préparation de l'intégration en EPS », Revue EP.S, n³03, p. 73-76.

${ }^{18}$ Pour un aperçu plus large sur le rôle des interactions, voir par exemple J. S. Bruner, Le développement de l'enfant. Savoir Faire, Savoir dire, Paris, PUF, 1983, particulièrement les chapitres 10 et 11, sur les interactions de tutelle et sur Vygotski. 


\section{1. Une communication favorisée}

Dans la mesure où certaines consignes peuvent être présentées par une démonstration des actions à effectuer, la compréhension est a priori moins difficile en EPS qu'ailleurs. Mais ce n'est pas le cas de toutes. Lorsqu'un élève n'a pas compris la consigne orale d'une tâche qui ne demande pas la reproduction d'un modèle gestuel, il risque d'imiter ses camarades et de s'interdire la recherche de ses propres solutions au problème posé (par exemple pour une activité d'opposition duelle ou collective).

Pour se faire comprendre, il importe que l'enseignant utilise des mots et une syntaxe suffisamment simples, ce qui ne signifie pas adopter un langage infantile. Il peut recourir à des reformulations, accompagner son message oral de gestes appropriés et ne pas s'en tenir à une consigne délivrée à l'ensemble du groupe. Notamment en situation d'intégration, la consigne collective a parfois besoin d'être reprise et adaptée à un élève en particulier. Par ailleurs, dans le cas d'un travail en ateliers, des dessins représentant ce qui est à faire peuvent être disposés pour chacun d'entre eux.

Les situations permettant à l'élève d'exprimer un choix sont recherchées, qu'il s'agisse de choisir un parcours à sa mesure, parmi d'autres de difficulté variable, de donner son point de vue sur la qualité d'une production lors d'une activité artistique, en l'argumentant dans la mesure du possible, etc. La prise de risque inhérente à l'expression personnelle mérite d'être valorisée, quelle que soit la pertinence de ce que qui est exprimé, car elle témoigne d'une confiance en soi favorable à l'autonomie.

\section{2. De l'auto-adaptation au guidage de l'activité}

Des recherches scientifiques, dont les résultats ont eu un fort écho chez les professeurs d'EPS, ont montré qu'un enseignement s'appuyant sur des démonstrations et des explications pour présenter aux élèves le comportement attendu est moins efficace qu'une stratégie consistant à les laisser rechercher la solution de façon autonome ${ }^{19}$. Dans le domaine des activités physiques adaptées aux personnes présentant un retard mental, les méthodes pédagogiques prônées vont généralement dans ce sens : elles «doivent valoriser les apprentissages qualifiés d'autoadaptatifs, c'est-à-dire réalisés à partir de milieux riches et aménagés. Dans ces situations d'apprentissage, le sujet s'auto-organise en fonction des sollicitations du milieu (...) et au regard du but de la tâche qui lui est proposé. Il construit donc sa propre motricité (...). L'apprentissage est ainsi vécu plus positivement en comparaison avec la pédagogie du modèle $»^{20}$.

Ces méthodes accordent une place de choix à un aménagement matériel de l'environnement conçu pour induire les comportements attendus. Par exemple, lors d'un déplacement en équilibre sur un banc, on peut obtenir un redressement du tronc et de la tête de l'élève en lui

\footnotetext{
${ }^{19}$ Famose J.-P. (1987), « Analyse et enseignement des habiletés motrices : l'intérêt de l'approche écologique », in Laurent M. et Therme P. (dir.), Apprentissage et développement des actions motrices complexes, Centre de recherche de l'UEREPS de Marseille, p . 343-358.

${ }^{20}$ Brunet F., Bui-Xuân G., Bluteau S., « Enseigner et animer les activités physiques adaptées aux personnes déficientes intellectuelles », in Varray A., Bilard J., Ninot G., Enseigner et animer les activités physiques adaptées, Dossier n55, Édit. Revue EP\&S, p. 140.
} 
demandant de toucher des ballons de baudruche suspendus au-dessus de lui à une hauteur adaptée, plutôt que de recourir à des consignes verbales. Le fait que l'élève puisse conduire son activité hors du regard de l'enseignant et qu'il prenne par lui-même connaissance du résultat atteint, à partir d'indices concrets issus de l'environnement, concourt à son autonomie.

L'intérêt de l'aménagement matériel ne doit toutefois pas conduire à méconnaître le rôle des interactions humaines dans l'apprentissage, notamment, dans le cadre des habiletés motrices, le rôle de la démonstration et des consignes d'action. Les travaux de Winnykamen et Lafont ${ }^{21}$, prolongeant ceux de Bandura, ont montré que l'observation d'autrui mobilisait des processus attentionnels, mnésiques, motivationnels et de reproduction motrice. Mais la pertinence des modalités de guidage dépend du type d'habileté à acquérir.

Quand il s'agit d'habiletés dites fermées (pour des tâches effectuées dans des conditions environnementales stables, sans incertitude : danse, gymnastique, tir à l'arc...) et dont le but est de reproduire des formes gestuelles, comme en danse, le pratiquant doit fixer un schème d'action conforme au modèle. À cet effet, la démonstration explicitée du geste s'avère supérieure à l'aménagement matériel. En revanche, dans le cas d'habiletés ouvertes, correspondant à des tâches réalisées dans un environnement incertain (l'incertitude pouvant tenir à l'environnement physique, dans des activités de pleine nature, ou humain, dans les sports d'opposition), la diversification des schèmes d'action et un guidage par la connaissance des résultats se révèlent efficaces. En fait, ce type d'habileté peut aussi faire appel à des gestes techniques. Pour traiter le problème posé par une tâche, plusieurs de ces gestes sont susceptibles d'être retenus, moyennant si besoin une adaptation personnalisée. L'apprentissage peut alors bénéficier d'une démonstration de techniques pouvant convenir à l'élève, ainsi que de consignes d'actions. Les procédures de guidage apparaissent complémentaires, selon la nature de la tâche, le contexte et les caractéristiques du sujet ${ }^{22}$.

\section{3. La sollicitation de la réflexion}

Il s'agit là d'accompagner le retour réflexif de l'élève sur son activité, c'est-à-dire de l'aider à réfléchir sur plusieurs de ses aspects : en évaluer le résultat, identifier la procédure utilisée pour l'obtenir, puis établir un lien entre procédure et résultat pour éventuellement s'y prendre autrement et progresser. Cette démarche relève d'un objectif de méthode, "apprendre à apprendre », qui conduit l'élève à trouver les modalités et les contextes d'action qui sont les plus pertinents pour lui : au basketball, il y a plus d'une technique pour tirer au panier, et une même technique peut être abordée différemment en fonction des obstacles rencontrés lors de l'apprentissage. Cet accompagnement de la réflexion permet aussi de relativiser un échec, pas en leurrant l'élève sur ce qu'il a réalisé, mais dans le sens où le résultat de son action peut être mis en relation avec la manière dont il a agi. Il est important que l'échec ne soit pas considéré comme une fatalité liée à sa personne et à son irréductible impuissance, mais à des façons de faire sur lesquelles il a prise. Certes, le retard mental est parfois si important que la démarche

\footnotetext{
${ }^{21}$ Winnikamen F., Lafont L., (1990), « Place de l'imitation-modélisation parmi les modalités relationnelles d'acquisition : le cas des habiletés motrices », Revue Française de Pédagogie, 92, p.23-30.

${ }^{22}$ Lafont L., « Entre démonstration et auto-régulation. Le rôle de procédures de guidage ajustées aux caractéristiques des apprenants : interaction de tutelle, imitation-modélisation interactive et coping modèles », 7e colloque européen sur l'auto-formation « Faciliter les apprentissages autonomes », ENFA, Toulouse, 18-19-20 mai 2006, Disponible sur : http://www.enfa.fr/autoformation/
} 
préconisée bute sur des limites infranchissables, mais croire a priori que la réflexion des élèves ne doit pas être sollicitée, en raison de leurs difficultés cognitives, participerait d'une représentation propre à les enfermer dans leurs manques.

Les adolescents surmontent d'autant mieux leurs difficultés que l'on s'attache à adapter la complexité des situations à analyser et les niveaux d'abstraction des messages. Dans la mesure du possible, le résultat d'une action doit être perceptible concrètement : nombre d'obstacles touchés, zone de réception matérialisée lors d'un saut en longueur, etc. Toutes les activités ne se prêtent pas à une telle objectivation. Celles qui se traduisent par la production de formes gestuelles sont plus délicates à apprécier. Si l'on veut inviter un élève à comparer deux mouvements, la question « est-ce que c'est pareil ? » risque d'être trop abstraite. Des questions plus précises peuvent $\mathrm{y}$ conduire progressivement en retenant des critères moins équivoques (« est-ce que $\mathrm{X}$ a les jambes pliées ? Et $\mathrm{Y}$ ? »). Ce type de question intermédiaire met l'élève en situation de réussite et s'inscrit dans « une démarche (qui) contribue à lever les facteurs de blocages (angoisse se transformant en mutisme, inhibition ou même en attitude agressive). $»^{23}$

\section{4. Les aides entre élèves et les soutiens spécialisés}

Le collège Pierre Mendès France illustre certains types d'aide que peuvent apporter des élèves « ordinaires » en EPS : en gymnastique, chaque groupe de travail comprend deux élèves de l'UPI, dont les autres ont la responsabilité, et, lors du jeu de thèque, la difficulté à se repérer dans l'espace est palliée par l'appariement de certains élèves de l'UPI avec des camarades de cinquième. Dans un autre collège, qui accueille en intégration individuelle quelques adolescents « handicapés », l'un d'eux désigne dans la classe d'accueil un camarade référent, volontaire, auquel il peut éventuellement recourir. Ailleurs, l'aide d'un élève « ordinaire » n'a lieu que ponctuellement, par exemple pour l'arbitrage.

Quant aux soutiens spécialisés, ils sont assurés par des adultes. Ce peut être un auxiliaire de vie scolaire, précieux notamment lors d'activités appelant une vigilance particulière, telle la natation. À Riom, c'est l'enseignant spécialisé qui seconde le professeur. Il intervient si besoin auprès d'autres élèves que ceux de l'UPI. Si, dans ce collège, les cours spécifiques aux élèves de l'UPI sont assurés par un professeur d'EPS, dans d'autres établissements c'est parfois l'enseignant spécialisé qui en est responsable. Dans l'un deux, il profite de ces cours pour préparer les élèves à des tâches qu'ils auront à réaliser en situation d'intégration, par exemple la direction d'un échauffement.

\section{5. Une juste distance}

L'autonomie nécessite de se déprendre d'un sentiment de compassion excessif qui conduit à aider les élèves au-delà du nécessaire, à les maintenir dans une dépendance qui peut d'autant plus perdurer qu'elle apporte des bénéfices aux deux parties qu'elle $l_{i e^{24}}$.

\footnotetext{
${ }^{23}$ Boyer J.-P., Markievich C., Galerin L., Julhien G. (dir.) (2002).), Accompagnement vers l'autonomie des élèves scolarisés dans une Unité Pédagogique d'Intégration, Document élaboré à la demande de la DESCO, Collège P. Mendès France, Riom .

${ }^{24}$ Memmi A. (1979), La dépendance, esquisse pour un portrait du dépendant, Paris, Gallimard.
} 
Concernant les relations entre élèves, l'étude conduite à Riom met en évidence une évolution au fil du temps : « Au début, remarque un professeur, il y avait un peu de distance, voire parfois quelques réticences » de la part des élèves « ordinaires » à l'égard de ces collégiens différents. «On ne se mélangeait pas. On a eu quelques difficultés à faire accepter ces séances communes ». Pour la représentante d'une association de parents d'élèves de l'établissement, qui a fait le même constat, les difficultés initiales s'expliquent par le fait que l'« on a des $a$ priori sur le handicap, le handicap fait un peu peur, et donc il faut apprendre à se connaître ». Est-ce qu'une intégration collective, donc la présence d'un nombre relativement élevé de jeunes de l'UPI au sein d'une classe ordinaire, ne serait pas plus délicate, pour les élèves de cette classe, que des intégrations individuelles ? Paradoxalement, selon ce parent, la distance initiale s'est conjuguée parfois avec une certaine surprotection des élèves ordinaires à l'égard des adolescents de l'UPI : « Au départ, c'était pour eux des enfants handicapés, qui savaient moins faire qu'eux, qu'il fallait protéger. Donc, ils étaient un peu derrière pour les aider ».

Progressivement, les relations entre élèves sont devenues plus équilibrées. Toutefois, un professeur d'EPS souligne que l'aide dont bénéficient les adolescents en situation de handicap doit être appréciée avec nuance. Si en effet elle présente à ses yeux des aspects très positifs, il arrive que des élèves de l'UPI lui paraissent moins autonomes en situation d'intégration : « Lors de certaines activités, que l'on a pu pratiquer uniquement avec eux, ils se débrouillent très bien sans l'aide de personne. Et quelquefois, lorsqu'ils sont en en contact avec ces élèves-là (du collège), d'un seul coup, on a l'impression qu'ils ne savent plus faire, parce qu'ils comptent sur eux alors qu'ils savent ». Mais cette attitude est conjoncturelle : dans d'autres tâches, selon une autre organisation, en particulier concernant les relations entre élèves, la réussite sera accrue.

Il arrive qu'un adolescent n'accepte d'agir qu'avec l'aide d'une personne en particulier, adulte ou camarade. Si un accompagnement personnalisé s'avère parfois nécessaire, il devrait, autant que possible, ne constituer qu'un détour sur le chemin de l'autonomie. S'efforcer de libérer l'activité de l'élève de son contexte initial est un objectif pédagogique qui déborde les aspects affectifs et relationnels. L'acquisition de compétences suppose en effet de pouvoir transférer ce qui a été appris dans une situation à une autre qui relève d'un même type de problème sans être exactement identique. Un joueur de football, par exemple, est appelé à mettre en œuvre des stratégies de démarquage dans des contextes de jeu qui ne sont jamais tout à fait les mêmes. Viser l'adaptabilité de l'élève est un objectif important, bien que difficile à atteindre avec des sujets qui peuvent avoir besoin d'un contexte d'activité très stable. Modifier un élément matériel utilisé pour structurer l'environnement peut être le premier pas d'une prise de distance vis-à-vis de repères rassurants, encore qu'un adolescent autiste puisse être davantage attaché à la stabilité de son environnement matériel qu'à la présence d'une personne déterminée. 


\section{Une construction permanente mais pas sans limites}

On peut considérer qu'un sujet est autonome lorsqu'il est « capable de se diriger tout seul, sans être contraint par quelqu'un d'autre à se conduire comme il le fait $»^{25}$. Mais le sens qu'il donne à ce qu'il désire et investit est en partie déterminé par celui qui a cours, inscrit dans les « significations imaginaires » de la société ${ }^{26}$ et dans les appartenances socioculturelles. Et les interactions avec autrui ne sont généralement pas dénuées de dépendance, plus ou moins selon les individus, les âges de la vie et les situations. Si l'autonomie apparaît donc être davantage un horizon qu'un état effectivement rencontré, son caractère relatif ${ }^{27}$ ne doit pas conduire à négliger le travail pour y tendre, notamment quand il concerne des personnes en situation de handicap.

Il s'agit bien d'un travail, entrepris par l'enseignant à partir d'un paradigme constructiviste, selon lequel le développement du sujet dépend largement de sa propre activité. Dans cette perspective, le but des tâches, l'adaptation de leurs conditions de réalisation, et le sens qu'elles peuvent présenter pour l'adolescent sont l'objet d'une vive attention, pour qu'il puisse et désire s'y engager en mobilisant toutes ses ressources, et pour qu'il progresse. Le sujet ne se construisant pas tout seul, l'interactionnisme est une autre référence propre à orienter le travail de l'enseignant. Les interactions entre les adultes et les élèves, et entre les élèves eux-mêmes, sont pensées de manière à solliciter et accompagner l'adolescent dans toutes ses dimensions : physique, cognitive, affective et relationnelle. Elles sont pour lui l'occasion d'une reconnaissance dont le besoin, «vital (...), constitue la validité nécessaire de la construction de soi $»^{28}$ et le socle de l'autonomisation.

L'importance accordée à l'autonomie nécessite de toujours veiller à la construire tout en étant conscient de ses limites. En y mettant l'accent, il importe tout d'abord de ne pas négliger le renforcement du lien social, auquel l'EPS peut apporter une contribution originale. De par les multiples interactions qu'elle suscite, elle constitue un support intéressant pour apprendre à vivre ensemble et incarner des aspects de l'éducation civique. Et elle ouvre à une participation sociale d'autant plus large que l'on délaisse, dans le cadre de son enseignement, les activités physiques de nature rééducative au bénéfice de pratiques culturelles, porteuses de significations mobilisatrices $^{29}$.

En second lieu, l'autonomisation ne doit pas être entendue comme antinomique avec des aides, même si parfois elles signent une dépendance. Certes, elles peuvent entraver l'autonomie, mais paradoxalement elles en sont une condition si elles ne s'opposent pas à la volonté du sujet et si elles lui permettent une activité qui sans cela serait impossible. On peut donc admettre qu' « est autonome celui qui sait demander et obtenir l'aide dont il a besoin pour élaborer ses projets et

\footnotetext{
${ }^{25}$ Descombes V. (2004), Le complément de sujet. Enquête sur le fait d'agir de soi-même, Paris, Gallimard, p. 443.

${ }^{26}$ Castoriadis C. (1975), L’institution imaginaire de la société, Paris, Éditions du Seuil, Coll. « Esprit ».

${ }^{27}$ Sticker H.-J. (1997), « L'autonomie, une valeur relative », in Trisomie : le défi de l'autonomie, Actes des 6es journées nationales Trisomie 21, Geist 21, Clermont-Ferrand.

${ }^{28}$ Gardou C. (1998). « La personne handicapée : d'objet à sujet, de l'intention à l'acte», Colloque, Lyon II, 17-

19 sept 1998, La nouvelle revue de l'AIS, Éd. du Cnefei, n 4, p. 99.

${ }^{29}$ Bruner J.S., (1996). L'éducation, entrée dans la culture, op. cit.
} 
les réaliser ${ }^{30}$. Ce qui suppose d'apprendre à connaître ses limites, à apprécier ce qui est réalisable seul ou non. De ce point de vue, l'EPS présente l'intérêt de placer l'élève dans des situations où la réussite et l'échec sont donnés à voir objectivement. La conscience de ses capacités était manifestement absente chez cette adolescente qui « est partie dans la partie profonde du bassin sans savoir nager ${ }^{31}$, à l'opposé de cette autre qui interpelle un camarade pour lui demander une aide justifiée en gymnastique.

Enfin, si l'autonomie d'un sujet en situation de handicap peut susciter parfois des résistances chez les enseignants et les éducateurs, en raison de l'incertitude dont elle est porteuse et des fonctionnements institutionnels routiniers qu'elle dérange, il arrive qu'elle soit promue jusqu'à devenir une injonction à laquelle la personne est incapable de répondre, courant le risque de l'instillation d'un sentiment d'échec durable, alors qu'une estime de soi suffisante est requise pour oser exprimer un choix, agir de soi-même, s'engager dans un projet et le mener à bien.

\section{Références bibliographiques}

- BARON M., BRUNET F., VERRET C. (2005), Gérer au quotidien son autonomie fonctionnelle. Activités physiques adaptées aux personnes en perte d'autonomie, Dossier EP.S $\mathrm{n}^{\circ} 63$, Éd. Revue EP\&S.

- BRUNET F. et MAUTUIT D. (2003), Activités physiques adaptées aux personnes déficientes intellectuelles. Du programme institutionnel au projet personnalisé, Dossier EP.S nº 60, Éd. Revue EP.S.

- BRUNET F. et BUI-XUAN G. (dir.), (1991), Handicap mental, troubles psychiques et sport, Afraps, Clermont-Ferrand, 1991.

- «Faciliter les apprentissages autonomes», Actes du 7e colloque européen sur l'autoformation, ENFA, Toulouse, 18-19-20 mai 2006, Disponible sur : http://www.enfa.fr/autoformation/.

- GAREL J.-P. (2001), Des collégiens parmi les autres. L'intégration en EPS d'élèves présentant un handicap mental, Doc. audiovisuel, 40', Suresnes, Édit. de l'INS HEA.

- GAREL J.-P. (2006), «EPS et élèves en situation de handicap : l'émancipation de la

${ }^{30}$ Celeste B. (1997), «L'autonomie, une demande paradoxale », in Trisomie : le défi de l'autonomie, op. cit., p.136.

${ }^{31}$ Témoignage de C. Chafiol sur : http://www.ac-orleans-

tours.fr/eps/eps_adaptee/UPI_CLG_NERUDA_St_Pierre_corps.pdf 
didactique ", La nouvelle revue de l'adaptation et de la scolarisation, $\mathrm{n}^{\circ} 33$, Édit. de l'INSHEA.

- GAREL J.-P. (2007), « L'autonomie du sujet déficient visuel : contribution de l'Éducation physique et sportive », in Sarralié C. (dir.) Scolariser les élèves déficients visuels aujourd'hui, La nouvelle revue de l'adaptation et de la scolarisation, Hors série $n^{\circ} 3$, Édit. de l'INS HEA, p.107-123.

- MEARD J.-A. et BERTONE S., L'autonomie de l'élève et l'intégration des règles en éducation physique, Paris, PUF, 1998.

- PIERRON J.-P. (2007), «L'autonomie dans la dépendance : le libre choix des personnes en situation de handicap », Bulletin d'informations du CREAI Bourgogne, $\mathrm{n}^{\circ}$ 266, p.10.

- STIKER, H.-J., GARDES P., KORFF-SAUSSE S. (et al) (1997), Trisomie : le défi de l'autonomie, Actes des 6èmes journées nationales Trisomie 21, Geist 21, Clermont-Ferrand.

- VARRAY A., BILARD J. et NINOT G. (dir.), (2001), Enseigner et animer les activités physiques adaptées, Dossier n55, Édit. Revue EP\&S. 